\title{
Seek \& Beautify: integrating UAVs in the optimal beautification of e-scooter sharing fleets
}

\author{
Stefano Carrese*, Fabio D’Andreagiovanni ${ }^{\dagger},{ }^{\ddagger}, \S$, Antonella Nardin*, Tommaso Giacchetti*, Leonardo Zamberlan* \\ *Department of Engineering, Università degli Studi Roma Tre, Rome, Italy \\ ${ }^{\dagger}$ French National Centre for Scientific Research (CNRS), France \\ ${ }_{\ddagger}^{\ddagger}$ Heudiasyc UMR 7253, Sorbonne Universités, Université de Technologie de Compiègne, CNRS, Compiegne, France \\ ${ }^{\S}$ Corresponding Author, email: d.andreagiovanni@hds.utc.fr
}

\begin{abstract}
Electric scooter (e-scooter) sharing has recently known a wide success in many cities all around the world. Nevertheless, it has also generated issues due to risky and improper behaviour of its users. Wild parking, namely parking without caring about the rules of the road, has in particular become a major issue and has induced an increasing number of cities to impose bans and fines to e-scooter sharing. To tackle wild parking, we introduced the figure of the beautificator, an agent hired by a sharing company with the specific task to reposition e-scooters for guaranteeing urban decorum. In this paper, we propose to increase the effectiveness of the beautificators by integrating Unmanned Aerial Vehicles (UAVs) in their activities: remotely controlled UAVs equipped with cameras are deployed to fly across the sharing service area for identifying e-scooters that require beautification with priority. Thanks to the UAVs, the beautificators do not have to operate blindly, touring locations of parked e-scooters without knowing their parking condition, but can readily learn which e-scooters require their immediate attention. We formulate the problem of optimally scheduling the joint actions of beautificators and UAVs, taking into account beautification constraints, battery limits of UAVs and the possibility of swapping exhausted UAV batteries. For tackling this problem, we propose a mixed integer programming model and a heuristic for accelerating the convergence to the optimum of a state-of-the-art optimization solver, reporting results of computational tests over realistic instances.

Index Terms-E-scooters; Shared Mobility; Micromobility;
\end{abstract} Urban Decorum; Optimization; Heuristics.

\section{INTRODUCTION}

In recent times, electric scooters (e-scooters) have become a major successful expression of micro and shared mobility and have widely spread across cities all around the world. As highlighted in studies like [13], [15], in some countries they have started to consistently replace walking and biking of individuals and have also induced a remarkable mode-shift from private car trips. The wide success that e-scooters have experienced can be traced back to major advantages that they offer, like low buying price and maintenance cost and the ease with which they can be driven and parked. Furthermore, they have been welcomed as a way to decrease pollution and traffic, representing a valid sustainable alternative to fossil fuel-based private cars. For a wider introduction to features and benefits

978-1-7281-8995-6/21/\$31.00 @2021 IEEE of e-scooter mobility and, more in general, to shared mobility, we address the reader to [5], [12], [14], [16].

Thanks to the big success of e-scooters, an increasing number of sharing companies has appeared in many cities around the world. However, the easiness of parking that characterizes such sharing services has soon started to constitute a major source of issues: users have shown a clear tendency to wild parking, namely parking without respecting road rules and abandoning vehicles in risky positions that may pose threats to pedestrians on sidewalks and to other vehicles on the streets. Due to wild parking, local governments have started to impose bans and fines to sharing companies (see e.g., [9]).

With the aim of tackling wild parking and not reducing acceptance of shared e-scooters by city residents, some companies have begun to consider the correction of wrongly parked scooters as a relevant part of their operations. Inspired by this, in [4] we have proposed to introduce the figure of the beautificators, agents that are hired by a sharing company expressly for contrasting wild parking and guaranteeing urban decorum. The critical task of a beautificator is to reposition e-scooters over short distances (even just a few meters), so as to fix inappropriate and disordered parking made by users. We stress that such repositioning must not be confounded with traditional relocation made in vehicle-sharing systems for rebalancing fleets in the service area. Relocation is indeed operated over medium and long distances in a city, with the primary objective of obtaining a balanced distribution of vehicles in the service area, so as to better satisfy user demand and increase profit (e.g., [3]).

In [4], inspired by and based on discussions that we had with professionals from a major European e-scooter sharing company, besides introducing the concept and figure of the beautificator, we identified the optimization problem of a sharing company that must optimally schedule the actions of its beautificators taking into account a number of operational constraints. We proposed an Integer Linear Programmingbased approach to represent and solve his problem, which, to the best of our knowledge was never addressed in literature.

In the present work, we propose to enhance the effectiveness and efficiency of the activities of the beautificators, by integrating the use of Unmanned Aerial Vehicles (UAVs - we refer the reader to [1], [17] for an introduction to UAVs and an overview 
of their applications in transportation engineering): remotely controlled UAVs equipped with cameras are deployed by the sharing company with the task of flying across the service area and identifying those scooters that requires beautification with priority (for example because they were parked on their side and may constitute a dangerous obstacle - see Figure 1). This intervention with priority is especially aimed at avoiding fines that could be imposed by patrolling police to the company for wild parking. Thanks to the assistance of the UAVs, beautificators do not operate blindly, but can more promptly intervene on those e-scooters that are more in need of beautification. Indeed, when the visual inspection of UAVs is not available, beautificators only know the position of the e-scooters and do not have accurate information about their parking status (e.g., whether they are parked in risky positions in the sidewalk ). Therefore, they would just randomly move from parked e-scooter to parked e-scooter without knowing which ones are really in need of beautification and could waste time to reach e-scooters that are already finely parked.

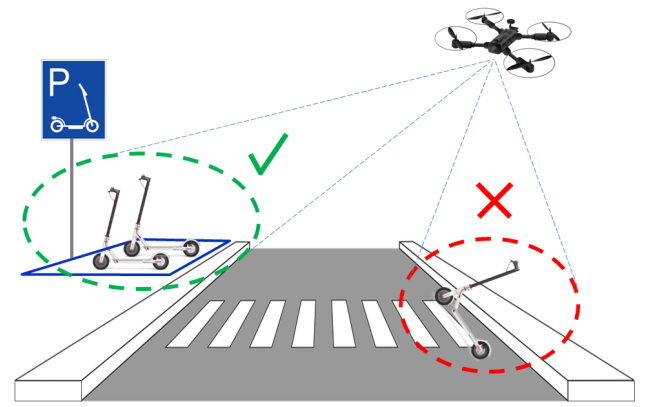

Fig. 1. Example of UAV checking e-scooter parking status

More in detail, we consider a sharing company that owns and manages a fleet of e-scooters with non-swappable batteries and that has at disposal a group of beautificators and a fleet of UAVs. The actions of the beautificators and UAVs are planned over a time horizon partitioned into a set of equal time slots, while the target area is decomposed into a set of zones. In each time slot, each beautificator may execute one among the following four types of actions: 1) "beautifying" the parking of one e-scooter in the zone where he/she is located, putting the e-scooter in a better position (e.g., if the e-scooter has fallen on its side, it is put into vertical position, while if it has been left in a position of the curb that interfere with pedestrian walk, it is moved to the side of the curb); 2) swapping a depleted battery of a UAV with a fully charged battery; 3) moving to another zone; 4) waiting in the zone where he/she is located. Each UAV, in each time slot, may instead execute one among the following four types of actions: 1) seeking escooters needing beautification with priority, by exploring the zone where it is located; 2) being subject to a battery swap by a beautificator; 3) moving to another zone; 4) waiting in the zone where it is located. Each action requires a number of time slots to be executed and is associated with a monetary value that jointly takes into account the cost and benefits of the action (in particular that of parking in line with urban decorum). The objective is to schedule the actions of the beautificators over the time horizon maximizing the total monetary value.

In this paper, our main original contributions are:

- proposing the integration of UAVs with beautification operations in e-scooter sharing, formulating the optimization problem of their joint optimal management;

- defining a mixed integer (linear) programming model for representing the problem, which includes an unsplittable multicommodity flow model as core;

- proposing a solution approach that includes a warm-start heuristic for accelerating the convergence to the optimum of a state-of-the-art optimization solver;

- reporting results of computational tests based on realistic instances that show the advantages of integrating UAVs to support the activities of the beautificators;

For modelling the addressed optimization problem, we rely on a multiperiod graph that possesses one node for each zonetime couple and in which arcs between nodes represent actions that can be executed by the beautificators and UAVs. The execution of actions is represented by means of an unsplittable multicommodity flow model, in which boolean flow variables model whether a beautificator or UAV does or does not execute an action and flow conservations constraints guarantee coherence of actions over space and time. Furthermore, additional constraints are included for modelling interactions between beautificators and UAVs, battery limitations of UAVs and movements between zones.

The remainder of this paper is organized as follows: in Section II, we introduce an optimization model for the joint optimization of UAVs and beautificators actions, while in Section III we present the adopted solution approach. Finally, we report results of computational tests in Section IV.

\section{Optimization MODEL}

As first modelling step, we decompose the e-scooter service area into a set of zones denoted by $Z$. In this area, the beautification is operated by a set of beautificators $B$ and supported by a set of UAVs $U$. The beautification takes place over a time horizon in which we identify a set of time instants $T=\left\{0,1, \ldots, t^{\max }\right\}$. Each couple of consecutive time instants of $T$ defines a time slot of duration $\Delta t$.

At the beginning of the time horizon (i.e., at $t=0$ ), each zone $z \in Z$ contains a number $n_{z}^{\text {TOT }} \geq 0$ of e-scooters. Among these e-scooters, a number $n_{z}^{\mathrm{PR}} \geq 0$ requires to be beautified with PRiority $(P R)$, whereas the remaining number $n_{z}^{\mathrm{NPR}}=$ $n_{z}^{\mathrm{TOT}}-n_{z}^{\mathrm{PR}}$ requires beautification with No-PRiority (NPR) (in what follows, we will simply refer to these e-scooters as either with priority or without priority). We assume that the decision maker does not know which are the $n_{z}^{\mathrm{PR}}$ e-scooters with priority and may identify them by using the UAVs as seekers that fly through a zone. In each time slot, we assume that one single UAV is able to reveal a fraction $\alpha_{z} \in[0,1]$ of the e-scooters with priority that are located in the zone $z$ explored by the UAV. Each UAV is equipped with a battery whose charge $c$ must lie in the range $\left[C^{\min }, C^{\max }\right]$ and is consumed by executing actions. For replenishing the charge, a 
beautificator may operate a battery swap, i.e. may substitute a battery with low charge with another battery charged at $C^{\max }$ (we thus assume that battery swapping is the only option for recharging the UAVs and that there are no charging stations where the UAVs may land).

In order to model the actions executed by the beautificators and the UAVs over time, we rely on a multicommodity flow model based on a graph $G(N, A)$ made up of:

- a set of nodes $N=Z \times T$ containing one node $(z, t)$ for each zone-time instant couple (with $z \in Z, t \in T$ );

- a set of arcs $A$ that is composed of the following six disjoint subsets, each representing one type of action that a beautificator or a UAV may execute:

\section{a) Subsets of arcs specific for the beautificators:}

1) $A^{\mathrm{BEAU}-\mathrm{NPR}}$ - set of non-priority beautification arcs $a=$ $\left[(z, t),\left(z, t+m^{\text {BEAU-NPR }}\right)\right]$ representing the action of beautifying an e-scooter without priority in zone $z$. Here, $m^{\text {BEAU-NPR }}$ is the number of time slots required to beautify a single e-scooter without priority. Each arc is associated with a profit coefficient $\pi_{a}>0$ that reflects the benefit of guaranteeing urban decorum and depends upon the zone where the beautification takes place;

2) $A^{\text {BEAU-PR }}$ - set of priority beautification arcs $a=$ $\left[(z, t),\left(z, t+m^{\mathrm{BEAU}-\mathrm{PR}}\right)\right]$ representing the action of beautifying an e-scooter with priority in zone $z$. Here, $m^{\text {BEAU-PR }}$ is the number of time slots required to beautify a single e-scooter with priority. Each arc is associated with a profit coefficient $\pi_{a}>0$ that reflects the higher benefit of correcting the parking of an e-scooter that requires beautification with priority and depends upon the zone where the beautification takes place (we note that, if considering the same zone, the value of $\pi_{a}$ for priority beautification is higher than that of non-priority beautification, reflecting the advantage of intervening on e-scooters that are more in need of beautification);

3) $A^{\text {MOVE-B }}$ - set of beautificator movement arcs $a=$ $\left[\left(z_{1}, t\right),\left(z_{2}, t+m_{z_{1} z_{2}}^{\text {MOVE-B }}\right)\right]$ representing the action of a beautificator who changes the zone where he/she is operating, specifically moving from zone $z_{1}$ to zone $z_{2}$. Here, $m_{z_{1} z_{2}}^{\text {MOVE-B }}$ is the number of time slots that are required to move from $z_{1}$ to $z_{2}$. This type of arc is associated with a profit $\pi_{a}<0$ that represents the cost of moving the beautificator;

\section{b) Subsets of arcs specific for the UAVs:}

1) $A^{\mathrm{SEEK}}$ - set of seek arcs $a=[(z, t),(z, t+1)]$ representing the action of a UAV that explores zone $z$ for one time slot with the aim of seeking e-scooters requiring beautification with priority. Each arc is associated with a null profit coefficient $\pi_{a}=0$ and a negative variation $\Delta c_{a}<0$ of the battery;

2) $A^{\mathrm{MOVE}-\mathrm{U}}$ - set of UAV movement arcs $a=$ $\left[\left(z_{1}, t\right),\left(z_{2}, t+m_{z_{1} z_{2}}^{\mathrm{MOVE}-\mathrm{U}}\right)\right]$ representing the action of a UAV who changes the zone where it is operating, specifically moving from zone $z_{1}$ to zone $z_{2}$. Here, $m_{z_{1} z_{2}}^{\mathrm{MOVE}-\mathrm{U}}$ is the number of time slots that are required to move from $z_{1}$ to $z_{2}$. This type of arc is associated with a null profit $\pi_{a}=0$ and a consumption $\Delta c_{a}<0$ of the battery. We note that we must distinguish between movements MOVE-B of beautificators and movements MOVE-U of UAVs, since a UAV is able to move faster than a beautificator between zones, thus defining a different set of arcs.

c) Subsets of arcs for both the beautificators and the UAVs:

1) $A^{\text {SWAP }}$ - set of battery swap arcs $a=[(z, t),(z, t+$ $\left.\left.m^{\text {SWAP }}\right)\right]$ representing the action of a beautificator who operates a battery swap on a UAV or the action of a UAV whose battery is swapped. This type of arc is associated with a profit $\pi_{a}<0$ reflecting the cost of battery swap. A swap fully replenish the charge of the UAV to $C^{\max }$;

2) $A^{\text {WAIT }}$ - set of waiting arcs $a=[(z, t),(z, t+1)]$ representing the action of either a beautificator or UAV that waits in a zone for one time slot. This type of arc is associated with a null profit $\pi_{a}=0$ and null UAV charge variation.

We define the subset of arcs: $A^{B}=A^{\text {BEAU-NPR }} \cup A^{\text {BEAU-PR }} \cup$ $A^{\text {MOVE-B }} \cup A^{\text {SWAP }} \cup A^{\text {WAIT }}$ to identify those arcs that may be used by the beautificators. Similarly, we define the subset $A^{U}=A^{\text {MOVE-U }} \cup A^{\mathrm{SEEK}} \cup A^{\mathrm{SWAP}} \cup A^{\text {WAIT }}$ to specifically refer to arcs that may be used by the UAVs. The overall set of arcs $A$ is thus equal to: $A=A^{B} \cup A^{U}$. Furthermore, given a node $(z, t) \in N$, we denote by $\delta^{\mathrm{FW}-\mathrm{U}}(z, t)\left(\delta^{\mathrm{FW}-\mathrm{B}}(z, t)\right)$ its forward (backward) star, i.e., the subset of arcs $a=[(z, t),(\bar{z}, \bar{t})] \in A$ having $(z, t)$ as tail (head) node.

Decision Variables. In order to model the actions of the beautificators and UAVs over time and space, we adopt a multicommodity flow model in which the actions are represented as flows moving through the graph $G(N, A)$. The model is based on the following families of decision variables:

- binary beautificator action variables $x_{a}^{b} \in\{0,1\} \forall b \in$ $B, a \in A^{B}$ such that:

$x_{a}^{b}= \begin{cases}1 & \text { if beautificator } b \text { executes the action } a \\ 0 & \text { otherwise }\end{cases}$

- binary UAV action variables $y_{a}^{u} \in\{0,1\} \forall u \in U, a \in A^{U}$ such that:

$$
y_{a}^{u}= \begin{cases}1 & \text { if UAV } u \text { executes the action } a \\ 0 & \text { otherwise }\end{cases}
$$

- continuous battery charge variables $c_{t}^{u} \in\left[C^{\min }, C^{\max }\right]$ (with $0 \leq C^{\min } \leq C^{\max }$ ) to represent the charge level of the battery of each UAV $u \in U$ at time $t \in T$;

- auxiliary integer variables $e_{t}^{z} \in \mathbb{Z}_{+}$to represent the number of e-scooters with priority that have been identified in zone $z$ by UAVs through SEEK actions until time $t$.

Feasibility Constraints. First, we need two sets of constraints to represent where the beautificators and UAVs start their actions. If we denote by $z_{0}(b) \in Z$ the zone where a beautificator $b$ is at time $t=0$ and by $z_{0}(u) \in Z$ the zone where a UAV $u$ is at time $t=0$, the constraints are:

$$
\sum_{a \in \delta^{\mathrm{FW}}(z, 0) \cap A^{B}} x_{a}^{b}=\left\{\begin{array}{ll}
1 & \text { if } z=z_{0}(b) \\
0 & \text { if } z \neq z_{0}(b)
\end{array} \forall z \in Z, b \in B\right.
$$


$\sum_{a \in \delta^{\mathrm{FW}}(z, 0) \cap A^{U}} y_{a}^{u}=\left\{\begin{array}{ll}1 & \text { if } z=z_{0}(u) \\ 0 & \text { if } z \neq z_{0}(u)\end{array} \forall z \in Z, u \in U\right.$

imposing that at $t=0$ every beautificator and UAV must choose exactly one of the actions of zone $z_{0}$ where it is initially located, while the actions of other zones must be set to zero.

The coherence of actions executed by the bautificators and UAVs over space and time in the graph $G(N, A)$ is guaranteed by the following flow conservations constraints:

$$
\begin{aligned}
& \sum_{a \in \delta^{\mathrm{BW}}(z, t) \cap A^{B}} x_{a}^{b}=\sum_{a \in \delta^{\mathrm{FW}}(z, t) \cap A^{B}} x_{a}^{b} \\
& \forall z \in Z, t \in T: 0<t<t^{\mathrm{max}}, b \in B \sum_{a \in \delta^{\mathrm{BW}}(z, t) \cap A^{U}} y_{a}^{u} \\
& \sum_{a \in \delta^{\mathrm{FW}}(z, t) \cap A^{U}} y_{a}^{u} \\
& \forall z \in Z, t \in T: 0<t<t^{\mathrm{max}}, u \in U
\end{aligned}
$$

The following constraints express that the number $e_{t}^{z}$ of e-scooters with priority identified until time $t$ in zone $z$ must equal the number of those identified until $t-1$ plus the number of those identified through SEEK actions executed from $t$ to $t-1$ in $z$ (we recall that every SEEK action of a UAV reveals $\alpha_{z} \cdot n_{z}^{N P R}$ e-scooters in $z$ ), namely:

$$
\begin{array}{r}
e_{t}^{z}=e_{t-1}^{z}+\alpha_{z} \cdot n_{z}^{N P R} \sum_{u \in U} y_{a}^{u} \quad \forall z \in Z, \\
t \in T: 1<t<t^{\max }, a \in A^{S E E K}: a=[(z, t-1),(z, t)]
\end{array}
$$

The previous number of identified e-scooters cannot exceed the total number of e-scooters with priority located in a zone $z$, so we must limit the number of SEEK actions that can be operated in $z$ over the entire time horizon:

$$
\sum_{u \in U} \sum_{a=[(\bar{z}, \bar{t}),(\bar{z}, \bar{t}+1)] \in A^{\mathrm{SEEK}}:} y_{a}^{u} \leq \frac{n_{z}^{\mathrm{NPR}}}{\alpha_{z}} \quad \forall z \in Z
$$

Concerning the battery charge of the UAVs, we must impose that at each time the charge level lies between the bounds and that at $t=0$ every UAV has maximum charge, namely:

$$
\begin{array}{r}
C^{\min } \leq c_{t}^{u} \leq C^{\max } \quad \forall u \in U, t \in T \\
c_{0}^{u}=C^{\max } \quad \forall u \in U
\end{array}
$$

Also, the charge of a UAV at each time instant $t$ must equal its charge at $t-1$ plus the (negative or positive) charge variation due to the action executed between $t-1$ and $t$ :

$$
\begin{aligned}
c_{t}^{u} & =c_{t-1}^{u}+\sum_{a \in A^{\mathrm{SEEK}} \cup A^{\mathrm{MOVE}-\mathrm{U}}} \Delta c_{a} \cdot y_{a}^{u}+ \\
& +\sum_{a \in A^{\mathrm{SWAP}}}\left(C^{\mathrm{max}}-c_{t-1}^{u}\right) \cdot y_{a}^{u} \forall u \in U, t \in T
\end{aligned}
$$

Note that, in the previous equality, the battery swap action brings the UAV to its maximum possible charge $C^{\max }$, filling the charge that is missing at $t-1$. Also, the non-linear product of variables can be easily linearized by standard techniques.

Finally, we must express the fact that the number of swaps of UAV batteries that can be operated in one time slot in a zone $z$ must not exceed the number of beautificators located in $z$ that may operate the swap, namely:

$$
\sum_{u \in U} y_{a}^{u} \leq \sum_{b \in B} x_{a}^{b} \quad \forall a \in A^{\mathrm{SWAP}}
$$

As finals step, we need to define two set of constraints to express the variable upper bound on the number of beautification with and without priority that can be executed in each time slot. First, we model the bound with priority:

$$
\begin{gathered}
\sum_{b \in B} x_{a}^{b} \leq e_{z}^{t}-\sum_{b \in B} \sum_{\substack{a=\left[(\bar{z}, \bar{t}),\left(\bar{z}, \bar{t}+m^{\mathrm{BEAU}}\right)\right] \in A^{\mathrm{BEAU}-\mathrm{PR}}: \\
\bar{z}=z \wedge \bar{t}<t}} x_{a}^{b} \\
\forall a=\left[(z, t),\left(z, t+m^{\mathrm{BEAU}}\right)\right] \in A^{\mathrm{BEAU}-\mathrm{PR}}
\end{gathered}
$$

where number of beautificators that can traverse each arc $a \in$ $A^{\text {BEAU-PR }}$ (left-hand-side of the inequality) must be less than or equal to the number $e_{z}^{t}$ of e-scooters with priority that have been identified by UAVs in zone $z$ until time $t$ (expressed by the integer variable $e_{z}^{t}$ ), minus the number of e-scooters with priority that have been beautified before $t$ (expressed by the summation of variables $x_{a}^{b}$ over arcs in $A^{\text {BEAU-PR }}$ that involve zone $z$ and start in instants $\bar{t}$ preceding $t$ ).

Then, we model the bound related to no priority:

$$
\begin{gathered}
\sum_{b \in B} x_{a}^{b} \leq n_{z}^{\mathrm{TOT}}-e_{z}^{t}-\sum_{b \in B} \sum_{\substack{a=\left[(\bar{z}, \bar{t}),\left(\bar{z}, \bar{t}+m^{\mathrm{BEAU}}\right)\right] \in A^{\mathrm{BEAU}-\mathrm{NPR}}: \\
\bar{z}=z \wedge \bar{t}<t}} x_{a}^{b} \\
\forall a=\left[(z, t),\left(z, t+m^{\mathrm{BEAU}}\right)\right] \in A^{\mathrm{BEAU}-\mathrm{NPR}}
\end{gathered}
$$

where the number of beautificators that can traverse each arc $a \in A^{\text {BEAU-NPR }}$ (left-hand-side of the inequality) must be not higher than the total number $n_{z}^{\text {TOT }}$ of e-scooters in $z$, minus those with priority that have been identified until time $t$ (expressed by the integer variable $e_{z}^{t}$ ), minus the beautification without priority that have been executed before $t$ (expressed by the summation of variables $x_{a}^{b}$ over arcs in $A^{\text {BEAU-NPR }}$ that involve zone $z$ and start in instants $\bar{t}$ preceding $t$ ).

Objective Function. The goal is to maximize the total profit that can be obtained by executing the set of actions at disposal of the beautificators and UAVs:

$$
\max \sum_{a \in A} \sum_{b \in B} \pi_{a} \cdot x_{a}^{b}+\sum_{a \in A} \sum_{u \in U} \pi_{a} \cdot y_{a}^{u}
$$

The overall optimization model, which we denote by UAVBEAU-OPT, is based on the defined decision variables, the constraints (3)-(14) - with linearized constraints (11) - and the objective function (15).

\section{SOlution APPROACH}

The optimization model UAV-BEAU-OPT presented in the previous section is a complex binary linear program that contains a consistent number of complicating coupling constraints. Since even a state-of-the-art optimization software like CPLEX [10] may prove slow and experience difficulties when attempting to solve the model for instances of practical interest, we propose a procedure to accelerate its convergence 
towards an optimal solution. Specifically, we propose a heuristic approach for fast identifying feasible solutions of good quality, which can be provided to CPLEX as initial solutions for warm starting the solution process.

For defining a warm-start heuristic, we can first note that a source of issues for the solver may be represented by the large number of arcs $A^{\mathrm{MOVE}-\mathrm{U}}$ and $A^{\mathrm{MOVE}-\mathrm{B}}$, which represent movements between zones and which can potentially start at each time slot, thus making the graph (very) dense. To tackle this issue, we could consider a relaxation of UAV-BEAU-OPT where the movement arcs are fully removed from the timespace graph: indeed, by removing these arcs, we guarantee the feasibility of solutions, while reducing the solution space. However, the full removal may result too limitative. As an alternative, we thus rely on a partial removal, according to which movements between zones can be executed only every $\tau \in \mathbb{Z}_{+}$time slots (for example, every 4 time slots). This simple trick allows to identify feasible solutions in a much faster way, especially when considering larger values of $\tau$.

In order to improve the solution found following this partial arc removal strategy, we also rely on the execution of an Integer Linear Programming (ILP)-based improvement heuristic. This heuristic corresponds with executing a large neighborhood search that follows the principles of an exact search, i.e. it is formulated as an ILP problem and is solved to the optimum by an exact solver like CPLEX (see e.g., [2]). An exact search takes into account the fact that, while solving the complete problem can be difficult even for a state-of-the-art solver like CPLEX, optimally solving a suitable subproblem may instead result much easier.

Specifically, the exact ILP-based search we adopt is based on RINS (Relaxation Induced Neighborhood Search), a wellknown effective and efficient search that exploits linear relaxations available for an optimization problem [11]. More formally, let $(\bar{x}, \bar{y})$ be a feasible solution of UAV-BEAUOPT found by partially removing movement arcs. Also, let $\left(x^{S L R}, y^{S L R}\right)$ be the optimal solution of the linear relaxation of UAV-BEAU-OPT (i.e., the model obtained removing the integrality requirements on the decision variables $x, y$ ) with added the valid inequalities found by CPLEX in the root node of its branch-and-bound tree when solving UAV-BEAUOPT. We denote such Strengthened Linear Relaxation by the acronym SLR. The modified RINS we propose to adopt considers a subproblem of UAV-BEAU-OPT that is obtained by setting a-priori the value of decision variables whose value in the incumbent integral solution $(\bar{x}, \bar{y})$ and in the optimal fractional solution of the strengthened linear relaxation $\left(x^{S L R}, y^{S L R}\right)$ is sufficiently close. More in detail, for a given parameter $\rho \in(0,1)$, the $\mathrm{i}$-th variable $(x, y)_{i}$ of the decision vector $(x, y)$ is such that:

$(x, y)_{i}= \begin{cases}1 & \text { if }(\bar{x}, \bar{y})_{i}=1 \wedge\left(x^{S L R}, y^{S L R}\right)_{i} \geq 1-\rho \\ 0 & \text { if }(\bar{x}, \bar{y})_{i}=0 \wedge\left(x^{S L R}, y^{S L R}\right)_{i} \leq \rho \\ \text { free } & \text { otherwise }\end{cases}$

The resulting problem with a subset of variables a-priori set to either 0 or 1 is then solved by CPLEX. The solution obtained from it is a feasible solution for the complete problem UAV-BEAU-OPT and thus can be passed as initial solution to CPLEX for faster solving UAV-BEAU-OPT.

\section{PRELIMINARY COMPUTATIONAL RESUlTS}

In order to evaluate the proposed optimization approach, we considered a set of realistic instances referring to the Italian city of Rome and defined in collaboration with professionals of e-scooter sharing mobility. All the instances refer to a district located in the downtown area of the Italian city of Rome. The district has been decomposed into 15 zones and hosts a fleet of 50 e-scooters. A group of 5 beautificators and a fleet of 3 UAVs are deployed in order to conduct beautification operations during the night, according to the actions specified in Section II. Given this setting, we considered 10 instances, each characterized by a different starting distribution and condition of parking of the e-scooters in the area. The actions of the beautificators and UAVs take place in an overnight shift lasting from 11:00 PM to 5:00 AM, which we decompose into a set of 36 time slots of 10 minutes duration each, thus defining a set of 37 time instants. The profit coefficients associated with arcs were defined taking into account the potential profit that an escooter may generate over one day, when located in a specific zone at the beginning of the day. E-scooters identified as in need of beautification with priority by UAVs are associated with higher profit coefficients, reflecting the higher chances of being rented once beautified and the benefit of correcting bad parking and improving urban decorum. Concerning the energy variations of UAVs, we referred to energy models adopted in [6], [6], [17]. The computational tests were run on a $2.70 \mathrm{GHz}$ Windows-based machine equipped with 8 GB of RAM, using CPLEX as optimization solver. We imposed a computational time limit of 3 hours for solving UAV-BEAU-OPT by means of CPLEX, warm-started with the solution provided by the heuristic described in Section III. In the construction heuristic, we set $\tau=12$ (movement arcs maintained every 12 times slots), while in the RINS-based search we set $\rho=0.1$.

We report the results of the computational tests that we conducted in Table I, where $I D$ denotes the instance identifier, $v_{\mathrm{UBO}}^{*}$ is the optimal value (in EUR) of UAV-BEAU-OPT, $v_{\text {noUAV }}^{*}$ is the optimal value (in EUR) of the model without UAVs (i.e., there are no UAVs that may operate SEEK actions and thus all e-scooters are assumed to require beautification without priority), and $\Delta v^{*} \%$ is the percentage increase that the optimal value $v_{\mathrm{UBO}}^{*}$ related to beautification with UAVs grants with respect to that $v_{\text {noUAV }}^{*}$ of beautification without UAVs. As evident from the table, the adoption of UAVs leads to a consistent profit advantage that equals about $17 \%$ on average and is over $20 \%$ in the case of instances like I 2 and I7.

In Figure 2, we provide a pie chart to visualize how the beautificators and UAVs spend their time budget, by reporting the average percentage of time devoted to each action over the considered 10 instances. The first fact that can be highlighted is that beautificators and UAVs spend much more than a half of their time budget executing their core actions, namely beautifying e-scooters (BEAU) and seeking e-scooters requiring 
TABLE I

COMPUTATIONAL RESULTS

\begin{tabular}{c|ccc}
\hline ID & $\begin{array}{c}v_{\text {UBO }}^{*} \\
\text { (CPLEX) }\end{array}$ & $\begin{array}{c}v_{\text {noUAV }}^{*} \\
\text { (MH-EScooter) }\end{array}$ & $\Delta v^{*} \%$ \\
\hline I1 & 232 & 272 & 17.3 \\
I2 & 256 & 311 & 21.5 \\
I3 & 241 & 276 & 14.8 \\
I4 & 296 & 330 & 11.6 \\
I5 & 260 & 307 & 18.2 \\
I6 & 283 & 331 & 17.1 \\
I7 & 279 & 345 & 23.8 \\
I8 & 249 & 281 & 13.0 \\
I9 & 271 & 304 & 12.4 \\
I10 & 237 & 281 & 18.7 \\
\hline
\end{tabular}

beautification with priority (SEEK). Indeed, BEAU is executed on average for $64 \%$ of the time by beautificators, while SEEK is executed for $62 \%$ of the time on average by UAVs. The second most executed action is represented by swapping batteries (SWAP), which is equal to about $20 \%$ for both UAVs and beautificators: the equality of the percentages is due to the fact that one swapping requires one beautificator to intervene on one UAV located in the same zone. Concerning movements between zones, we note that beautificators spend consistently more time executing MOVE actions (18\% versus 9\%). This is due to the fact that beautificators need to move more often than UAVs, with the aim of reaching UAVs located in other zones and in need of a battery swap and with the aim of reaching more profitable zones characterized by higher concentrations of e-scooters requiring beautification with priority. It is also interesting to note that, while the beautificators are always acting and never execute WAIT actions, in contrast the UAVs spend a non-negligible amount of time waiting (about 7\%). This is due to the fact that UAVs may need a battery swap while operating in zones where a beautificator is not present. In this case, a UAV, before totally running out of battery with the risk of crashing, must land and then wait for a beautificator to come to its zone and swap its battery before returning active.

As future work, our plan is to extend the computational tests to instances of larger size, including multiple districts simultaneously. Since these instances are going to prove harder to solve, we also intend to develop refined solutions approaches based on identifying mathematically stronger versions of the proposed model and developing matheuristics integrating exact and heuristic optimization methods. Furthermore, we intend to include considerations about e-scooters trip times, adapting and improving approaches such as [8], and investigate how trip time may result correlated to wild parking habits of users.

\section{REFERENCES}

[1] E.N. Barmpounakis, E.I. Vlahogianni, J.C. Golias, "Unmanned Aerial Aircraft Systems for transportation engineering: Current practice and future challenges", International Journal of Transportation Science and Technology, vol. 5, pp. 111-122, 2016.

[2] C. Blum, J. Puchinger, G.R. Raidl, A. Roli, "Hybrid metaheuristics in combinatorial optimization: A survey", Applied Soft Computing vol. 11, no. 6 , pp. 4135-4151, 2011.

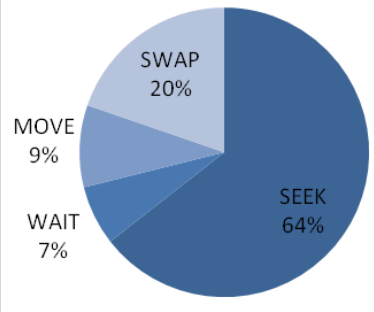

(a) UAVs

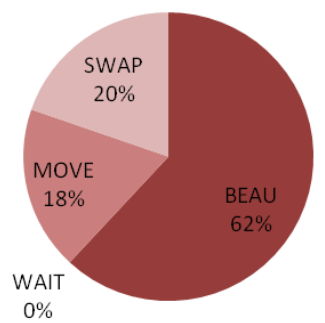

(b) Beautificators
Fig. 2. Average percentage of time spent by UAVs and beautificators on each of their feasible action types.

[3] B. Boyaci, K.G Zografos, N. Geroliminis, "An integrated optimizationsimulation framework for vehicle and personnel relocations of electric carsharing systems with reservations", Transportation Research Part B vol. 95, pp. 214-237, 2017.

[4] S. Carrese, F. D'Andreagiovanni, T. Giacchetti, A. Nardin, L. Zamberlan, "A Beautiful Fleet: Optimal Repositioning in E-scooter Sharing Systems for Urban Decorum", Transportation Research Procedia vol. 52, pp. 581-588, 2021.

[5] S. Carrese, F. D'Andreagiovanni, T. Giacchetti, A. Nardin, L. Zamberlan, "An optimization model and genetic-based matheuristic for parking slot rent optimization to carsharing", Research in Transportation Economics, vol. $85,2021$.

[6] L. Chiaraviglio, F. D’Andreagiovanni, W. Liu, J. Gutierrez, N. BlefariMelazzi, K.R. Choo, M. Alouini, "Multi-Area Throughput and Energy Optimization of UAV-Aided Cellular Networks Powered by Solar Panels and Grid", IEEE Transactions on Mobile Computing, DOI: 10.1109/TMC.2020.2980834, 2020.

[7] L. Chiaraviglio, F. D'Andreagiovanni, R. Choo, F. Cuomo and S. Colonnese, "Joint Optimization of Area Throughput and Grid-Connected Microgeneration in UAV-Based Mobile Networks", IEEE Access, vol. 7 , pp. 69545-69558, 2019.

[8] E. Cipriani, S. Gori, L. Mannini, S. Brinchi, "A procedure for urban route travel time forecast based on advanced traffic data: Case study of Rome", 17th International IEEE Conference on Intelligent Transportation Systems (ITSC), pp. 936-941, 2014

[9] CNN. 2019. "E-scooters suddenly appeared everywhere, but now they're riding into serious trouble". https://edition.cnn.com/travel/article/electric-scooter-bans-world

[10] IBM ILOG CPLEX: https://www.ibm.com/products/ilog-cplexoptimization-studio

[11] E. Danna, E. Rothberg, C. Le Pape, "Exploring relaxation induced neighborhoods to improve MIP solutions". Mathematical Programming 102, 71-90, 2005

[12] S. Gössling, "Integrating e-scooters in urban transportation: Problems, policies, and the prospect of system change". Transportation Research Part $D$ vol. 79, pp. 1-12, 2020.

[13] B. Laa, U. Leth, "Survey of E-scooter users in Vienna: Who they are and how they ride". Journal of Transport Geography vol. 89, 2020.

[14] C.A.S. Machado, N.P.M. De Salles Hue, F.T. Berssaneti, J.A. Quintanilha, "An Overview of Shared Mobility". Sustainability vol. 10, 2018

[15] R.L. Sanders, M. Branion-Calles, T.A. Nelson, "To scoot or not to scoot: Findings from a recent survey about the benefits and barriers of using E-scooters for riders and non-riders". Transportation Research Part A vol. 139 , pp. 217-227, 2020.

[16] S. Shaheen, A. Cohen, N. Chan, A. Bansal, "Sharing strategies: carsharing, shared micromobility (bikesharing and scooter sharing), transportation network companies, microtransit, and other innovative mobility modes". In "Transportation, Land Use, and Environmental Planning". E. Deakin (Ed.), Elsevier, Amsterdam, pp. 237-262, 2020.

[17] A. Trotta, F. D'Andreagiovanni, M. Di Felice, E. Natalizio, K.R. Chowdhury, "When UAVs Ride A Bus: Towards Energy-efficient Cityscale Video Surveillance", IEEE INFOCOM 2018, pp. 1043-1051, 2018. 